\title{
Impacto da segunda e terceira etapas do método canguru: do nascimento ao sexto mês
}

\author{
Impact of the second and third stages of the kangaroo method: from birth to sixth month \\ Impacto de la segunda y tercera etapas del método canguro: del nacimiento al sexto mes
}

\section{RESUMO}

Objetivos: Avaliar se o Método Canguru tem impacto nas taxas de aleitamento materno exclusivo, peso, tempo de internação e taxas de reinternação. Método: Trata-se de uma coorte retrospectiva, que incluiu recém-nascidos pré-termo de muito baixo peso ao nascer, os quais foram divididos em dois grupos: GCCo - composto por aqueles assistidos na Unidade de Cuidados Intermediários Neonatal Convencionais; GCCa composto por aqueles que foram assistidos na Unidade de Cuidados Intermediários Neonatal Canguru. Resultados: O grupo GCCa apresentou resultados superiores nas taxas de aleitamento materno exclusivo, no momento da alta hospitalar, primeira consulta ambulatorial, quarto mês de idade gestacional corrigida, além de menores taxas de reinternação. Conclusão: As segunda e terceira etapas do Método Canguru favoreceram a prática e manutenção do aleitamento materno exclusivo, além de apresentarem menores taxas de reinternação até o sexto mês de idade gestacional corrigida.

Descritores: Método Canguru; Prematuridade; Políticas públicas de saúde; Enfermagem.

\section{ABSTRACT}

Purpose: To evaluate whether the Kangaroo Method would have an impact on the rates of exclusive breastfeeding, weight, length of hospital stay and rates of hospital readmission. Methods: This is a retrospective cohort, which included very-low-weight preterm newborns. These were divided into two groups: GCCo - composed of those assisted in the Conventional Neonatal Intermediate Care Unit; GCCa - composed of those who were assisted at the Kangaroo Neonatal Intermediate Care Unit. Results: The GCCa group showed better results in exclusive breastfeeding rates at the time of hospital discharge and during outpatient follow-up - first outpatient consultation corrected fourth month of gestational age, in addition to lower rates of readmission. Conclusion: The second and third stages of the Kangaroo Method favored the practice and maintenance of exclusive breastfeeding, in addition to presenting lower rates of readmission until the sixth month of corrected gestational age

Descriptors: Kangaroo-Mother Care Method; Infant, Premature; Public health policies; Nursing.

\section{RESUMEN}

Objetivos: Evaluar si el Método Canguro tendría un impacto en las tasas de lactancia materna exclusiva, peso, tiempo de estancia hospitalaria y tasas de reingreso. Método: Se trata de un grupo retrospectivo, que incluyó a recién nacidos prematuros de muy bajo peso al nacer. Estos se dividieron en dos grupos: GCCo - compuesto por los atendidos en la Unidad de Cuidados Intermedios Neonatales Convencionales; GCCa - compuesto por los que fueron atendidos en la Unidad de Cuidados Intermedios Neonatales Canguro. Resultados: El grupo GCCa mostró resultados superiores en las tasas de lactancia materna exclusiva al momento del alta hospitalaria y durante el seguimiento ambulatorio - primera consulta ambulatoria en el cuarto mes de edad gestacional corregida, además de menores tasas de reingreso. Conclusión: La segunda y tercera etapa del Método Canguro favoreció la práctica y la prolongación de la lactancia materna exclusiva, además de presentar menores tasas de reingreso hasta el sexto mes de edad gestacional corregida.

Descriptores: Método Madre-Canguro; Prematuro; Políticas Públicas de Salud, Enfermería.
Fernanda Nascimento Alves ${ }^{1}$

0000-0003-0447-4230

Paula Carolina Bejo Wolkers ${ }^{1}$

D 0000-0001-8265-198X

Lucio Borges de Araújo ${ }^{1}$

D0000-0002-2230-203X

Daniela Marques de Lima

Mota Ferreira ${ }^{1}$

0000-0003-1378-3051

Vivian Mara Gonçalves de

Oliveira Azevedo ${ }^{1}$

0000-0002-7514-1508

1 Universidade Federal de Uberlândia.

Autor correspondente:

Fernanda Nascimento Alves

E-mail: fer.alves-1993@hotmail.com

\section{Como citar este artigo:}

Alves FN, Wolkers PCB, Ferreira DMLM et al. Impacto da segunda e terceira etapas do método canguru: do nascimento ao sexto mês. Revista de Enfermagem do CentroOeste Mineiro. 2021;11:e4200. [Access__]; Available in:__. DOI: http://doi.org/10.19175/recom.v11i0.4200 


\section{INTRODUÇÃO}

A prematuridade ainda é um desafio para o cuidado perinatal em todo o mundo. Os avanços tecnológicos e o melhor manejo nos cuidados da gestação e do neonato têm proporcionado aumento da sobrevida de recém-nascidos prétermo e de muito baixo peso (RNPTMBP). Entretanto RNPTMBP são mais predispostos, em relação aos nascidos a termo, a agravos como enterocolite necrosante, displasia broncopulmonar e sepse tardia ${ }^{(1)}$. Somado a isso, fatores como tempo prolongado de internação e inúmeros procedimentos invasivos podem ocasionar diversos desfechos clínicos desfavoráveis, sendo que as consequências, muitas vezes, ultrapassam 0 período perinatal ${ }^{(2)}$. Desmame precoce, dificuldade no crescimento somático e maiores taxas de reinternação estão entre os principais desafios encontrados após a alta hospitalar ${ }^{(3)}$.

Logo é crescente o estímulo à prática de medidas, a fim de atenuar os efeitos deletérios e, muitas vezes iatrogênicos, causados pelo ambiente hospitalar e procedimentos invasivos necessários à manutenção da vida do neonato enfermo. Assim, o foco da assistência neonatal passa por uma importante mudança de paradigma, cujo objetivo deixa de ser somente a sobrevivência, mas também o desafio de proporcionar um cuidado centrado no melhor desenvolvimento do RNPTMBP, capaz de devolver à família e à sociedade uma criança apta a desempenhar de maneira plena, suas capacidades físicas, afetivas e intelectuais.

Neste contexto, foi lançado o Método Canguru (MC), no Brasil, como política pública de saúde, cujo objetivo principal é desenvolver ações que favoreçam o cuidado centrado na família, a redução de fatores estressores ao RNPTBP, o aumento do aleitamento materno e o vínculo mãefilho-família( ${ }^{(4)}$. O MC é dividido em três etapas, sendo a primeira realizada dentro das Unidades de Terapia Intensiva Neonatal (UTIN), com ênfase na ambientação e acolhimento da família, estímulo à formação de vínculo por meio do toque e progressivamente com o posicionamento canguru, além de medidas protetoras para o RN, quanto ao estresse e dor. Já a segunda etapa se dá com o acompanhamento contínuo da mãe junto ao filho, nas Unidades de Cuidado Intermediário Neonatal Canguru (UCINca), em que também é realizado o contato pele a pele pela posição canguru, além de intensificar o estabelecimento do aleitamento materno e rotinas de cuidado com o recémnascido. A terceira etapa ocorre, após a alta hospitalar e consiste no acompanhamento ambulatorial até o RNPTBP atingir 2500g e condições de acompanhamento nos ambulatórios de seguimento(4).

Aqueles RNPTMBP cujas mães não têm possibilidade e/ou interesse em acompanhá-los continuamente, na segunda etapa do MC, seguem nas Unidades de Cuidado Intermediário Convencionais (UCINCo), recebendo todos os cuidados oferecidos, na primeira etapa do $M C$, porém sem a presença materna de forma contínua, o que acarreta, em consequência, redução na frequência de amamentação diretamente em seio, além de menores habilidades nos cuidados com o RN. Após a alta hospitalar, serão acompanhados em ambulatórios de seguimento e/ou nas redes de atenção básica da estratégia saúde da família( ${ }^{(4)}$.

O MC refere-se a uma política pública de saúde brasileira, que engloba diversos pilares, entre eles, o posicionamento canguru ou contato pele a pele. Apesar dos benefícios já comprovados em estudos realizados, principalmente no contexto internacional, como favorecimento do aleitamento materno ${ }^{(5)}$, ganho de peso ${ }^{(6)}$, redução no tempo de internação $0^{(7)}$ e menor risco de morte ${ }^{(8)}$, o posicionamento canguru trata-se apenas de um fundamento dentro de uma estratégia bem mais abrangente. Questiona-se então se o $M C$ seria capaz de proporcionar também outros benefícios, pois engloba uma pluralidade de ações e diretrizes que tem como intuito proporcionar assistência integral e humanizada ao RNPTBP e à sua família. Assim, o objetivo deste estudo foi avaliar se as segunda e terceira etapas do MC teriam impacto, nas variáveis clínicas neonatais, especificamente nas taxas de AME, no ganho ponderal, tempo de internação e taxas de reinternação, do nascimento ao sexto mês de idade gestacional corrigida (IGC).

\section{MÉTODOS}

A pesquisa foi realizada no serviço de neonatologia de um hospital universitário em Minas Gerais - Brasil, especificamente na unidade neonatal. Essa unidade possui 41 leitos, sendo 15 leitos destinados à UTIN, 26 à UCINCo e seis à UCINCa. A UCINCa, implementada em junho de 2016, recebe egressos da UTIN e UCINCo, conforme os critérios de elegibilidade, definidos pelo Manual Técnico do Ministério da Saúde(2). Esses critérios são divididos: critérios relacionados ao RN, que são a estabilidade clínica, nutrição 
enteral plena e peso mínimo de 1.250 gramas e critérios relacionados à mãe, que se tratado desejo de participar, disponibilidade de tempo e de rede social de apoio, consenso entre mãe, familiares e profissionais de saúde, capacidade de reconhecer os sinais de estresse e as situações de risco do RN e conhecimento e habilidade para manejar o RNPT em posição canguru.

Tratou-se de um estudo do tipo coorte retrospectivo. Os dados foram obtidos, por meio de análise de prontuário, em que se avaliou o tempo de internação, as taxas de amamentação, o peso no momento da alta hospitalar, tempo de aleitamento materno exclusivo pós-alta (primeira consulta ambulatorial, quarto e sexto mês de IGC) e as taxas de reinternação até o sexto mês de IGC.

A pesquisa foi submetida ao Comitê de Ética em Pesquisa e, pelo desenho de estudo retrospectivo, o Termo de Consentimento Livre e Esclarecido foi dispensado, ficando os autores responsáveis pelo sigilo das informações e não identificação dos participantes (aprovação número: 2521553).

Foram incluídos no estudo os RNPTMBP $(<1500$ g) de qualquer idade gestacional e excluídos os que evoluíram para óbito, necessitaram de transferência para outras instituições, apresentavam malformações congênitas ou alterações neurológicas sintomáticas que impossibilitavam ou desfavoreciam a realização do posicionamento canguru e da amamentação.

Os RNPTMBP incluídos foram divididos em dois grupos: Grupo de Cuidados Convencionais (GCCO) - composto por RNPTMBP que nasceram no período de 10 de janeiro a 31 de dezembro de 2015, com critérios de elegibilidade para UCINCa, mas que permaneceram na UCINCo, pois nesse período a UCINCa ainda não estava em funcionamento; Grupo de Cuidado Canguru (GCCa), composto por RNPTMBP, assistidos na UCINCa, que nasceram no período de 10 junho de 2016 a 31 de dezembro de 2017.

Considerando a média anual de nascimentos de RNPTMBP, nos dois anos de avaliação deste estudo na instituição e um nível de confiança de $95 \%$, estabeleceu-se, após cálculo amostral, que a amostra seria composta por, no mínimo, 63 prontuários. No entanto como se tratou de uma pesquisa retrospectiva, na qual há possibilidade de perdas de informações, optou-se por avaliar todos os prontuários dos RNPTMBP com critérios de elegibilidade, no período pré-estabelecido, totalizando 93 crianças incluídas.
Figura 1 - Fluxograma de inclusões e exclusões.

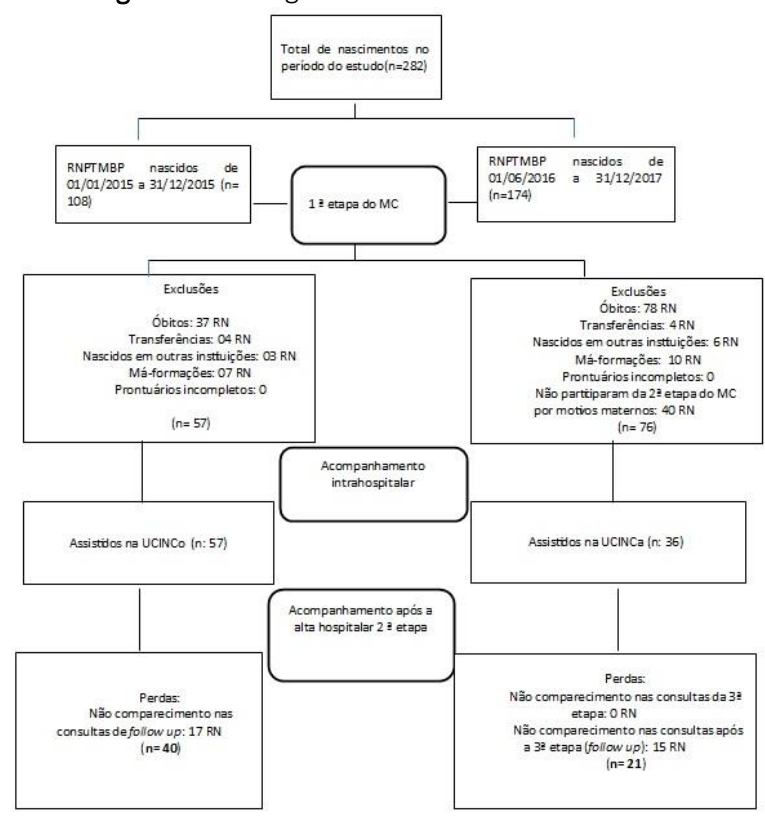

Fonte: Elaborado pelos autores (2021).

Legenda: RNPTMBP: recém-nascido pré-termo de muito baixo peso, RN: recém-nascido, MC: Método Canguru, UCINCo: Unidade de Cuidados Intermediários Neonatais Convencionais, UCINCa: Unidade de Cuidados Intermediários Neonatais Canguru.

Os RNPTMBP inclusos, em ambos os grupos, foram avaliados quanto às variáveis relacionadas ao parto (escore de Apgar de 50 minuto, peso de nascimento, IG ao nascimento), variáveis relacionadas à internação (tempo de ventilação mecânica, tempo total de oxigenioterapia, tempo de nutrição parenteral, dia de início de via enteral, dia de via enteral plena, menor peso, dia de início de amamentação, tempo de internação, peso de alta, tipo de alimentação no momento da alta) e variáveis de acompanhamento ambulatorial (tipo de alimentação na primeira consulta, 4ㅇ e 6ㅇ mês de IGC, peso no 4ㅇ e 60 mês de IGC, taxas de reinternação até o 60 mês de IGC). Destacamos que a primeira consulta ambulatorial referiu-se à consulta realizada, no ambulatório de follow up (comum a todos os RNPTMBP nascidos na instituição de estudo, tanto para os RNPTMBP que receberam alta da terceira etapa do $M C$, quanto para os que permaneceram somente na UCINCo).

As variáveis quantitativas foram descritas em médias, medianas, desvios-padrão e valores máximo e mínimo. Além disso, aplicou-se o teste de normalidade Shapiro-Wilk. Para as variáveis que apresentaram distribuição normal, aplicou-se o tStudent para a comparação dos grupos, caso contrário, foi aplicado o teste Mann-Whitney. As variáveis qualitativas foram descritas em frequência e porcentagem. As associações das 
variáveis qualitativas foram avaliadas por meio do teste razão de verossimilhança. Para avaliação dos desfechos, utilizou-se a regressão logística e logística ajustada para o peso de alta e IGC. Todos os testes foram aplicados considerando um nível de significância de $5 \% \quad(p<0.05)$. Os procedimentos foram realizados utilizando 0 software SPSS v. 20 .

\section{RESULTADOS}

Um total de 93 prontuários foram avaliados, sendo 57 pertencentes ao GCCo e 36 ao GCCa. Na Tabela 1 estão representadas as principais características clínicas dos RNPT incluídos no estudo.

Tabela 1 - Caracterização da amostra quanto às variáveis numéricas dos RNPTMBP e mães incluídas no estudo

\begin{tabular}{|c|c|c|c|}
\hline Variáveis & GCCo & GCCa & p-valor \\
\hline Idade materna (anos) ${ }^{\dagger}$ & $25,82 \pm 6,85$ & $25,92 \pm 5,71$ & 0,94 \\
\hline Idade gestacional (semanas) † & $28,98 \pm 2,17$ & $28,86 \pm 2,10$ & 0,79 \\
\hline Peso de nascimento ${ }^{\dagger}$ & $1082,79 \pm 238,48$ & $1069,67 \pm 226,56$ & 0,79 \\
\hline Apgar 5 o minuto * & $8,0(4,0-10,0)$ & $9,0(6,0-10,0)$ & 0,19 \\
\hline Tempo de ventilação mecânica (dias) * & $1,0(0,0-28,0)$ & $1,50(0,0-50,0)$ & 0,48 \\
\hline Tempo total de oxigenioterapia (dias) * & $20,0(1,0-95,0)$ & $24,0(0,0-50,0)$ & 0,83 \\
\hline Tempo de nutrição parenteral (dias) * & $13,0(1,0-37,0)$ & $14,50(0,0-52,0)$ & 0,50 \\
\hline Dia de início de via enteral (dias) * & $2,0(1,0-11,0)$ & $2,0(1,0-30,0)$ & 0,13 \\
\hline Dia de via enteral plena (dias) ${ }^{*}$ & $18,0(7,0-40,0)$ & $16,0(5,0-56,0)$ & 0,55 \\
\hline Menor peso (gramas) + & $909,68 \pm 229,06$ & $907,36 \pm 233,91$ & 0,96 \\
\hline Dia de início de amamentação (dias) † & $28,48 \pm 18,80$ & $23,47 \pm 12,67$ & 0,12 \\
\hline Duração da internação inicial (dias) † & $66,30 \pm 21,43$ & $61,06 \pm 18,39$ & 0,30 \\
\hline IGC na alta hospitalar (semanas) † & $38,14 \pm 2,55$ & $37,83 \pm 1,95$ & 0,53 \\
\hline Peso de Alta * & $2120,0(1915,0-3510,0)$ & $2027,5(1820,0-2730,0)$ & 0,00 \\
\hline Peso no Quarto mês de IGC† ${ }^{+}$ & $5959,75 \pm 1079,19$ & $5573,15 \pm 803,29$ & 0,11 \\
\hline Peso no Sexto mês de IGC * & $6980,0(4400,0-10790,0)$ & $6445,0(5130,0-9070,0)$ & 0,12 \\
\hline
\end{tabular}

Fonte: Elaborado pelos autores (2021).

Legenda IGC: idade gestacional corrigida, *: valores expressos em mediana (mínimo-máximo), † : valores expressos em média \pm desviopadrão.

As taxas de AME foram superiores no momento da alta, na primeira consulta de acompanhamento ambulatorial e quarto mês de IGC no grupo GCCa e as taxas de reinternação foram superiores no grupo GCCo. Além disso, houve menor número de retorno na primeira consulta de acompanhamento ambulatorial no grupo GCCa, após a alta da terceira etapa (Tabela 2).

Tabela 2 - Características clínicas na alta hospitalar e durante o acompanhamento ambulatorial dos RNPTMBP incluídos no estudo

\begin{tabular}{|c|c|c|c|c|c|c|c|}
\hline Variáveis & Momentos & Ocorrência & $\begin{array}{c}n \\
(\mathrm{GCCO})\end{array}$ & $\begin{array}{l}\text { Porcentagem } \\
\text { (GCCo) }\end{array}$ & $\begin{array}{c}n \\
\text { (GCCa) }\end{array}$ & $\begin{array}{l}\text { Porcentagem } \\
\text { (GCCa) }\end{array}$ & $\mathrm{p}$-valor \\
\hline \multirow{8}{*}{ AME } & \multirow{2}{*}{ Alta } & Sim & 1 & 1,8 & 6 & 16,7 & \multirow{2}{*}{0,00} \\
\hline & & Não & 56 & 98,2 & 30 & 83,3 & \\
\hline & \multirow{2}{*}{ Primeira consulta } & Sim & 1 & 1,9 & 5 & 21,7 & \multirow{2}{*}{0,00} \\
\hline & & Não & 52 & 98,1 & 18 & 78,3 & \\
\hline & \multirow{2}{*}{ Quarto mês de IGC } & Sim & 0 & 0,0 & 7 & 25,9 & \multirow{2}{*}{0,00} \\
\hline & & Não & 40 & 100 & 20 & 74,1 & \\
\hline & \multirow[t]{2}{*}{ Sexto mês de IGC } & $\operatorname{Sim}$ & 0 & 0,0 & 2 & 9,5 & \multirow[t]{2}{*}{0,36} \\
\hline & & Não & 40 & 100 & 19 & 90,5 & \\
\hline \multirow{8}{*}{ AMM } & \multirow{2}{*}{ Alta } & Sim & 28 & 49,1 & 26 & 72,2 & \multirow{2}{*}{0,026} \\
\hline & & Não & 29 & 50,9 & 10 & 27,8 & \\
\hline & \multirow{2}{*}{ Primeira consulta } & Sim & 26 & 49,1 & 11 & 47,8 & \multirow{2}{*}{0,921} \\
\hline & & Não & 27 & 50,9 & 12 & 52,2 & \\
\hline & \multirow{2}{*}{ Quarto mês de IGC } & Sim & 9 & 22,5 & 7 & 25,9 & \multirow{2}{*}{0,748} \\
\hline & & Não & 31 & 77,5 & 20 & 74,1 & \\
\hline & \multirow{2}{*}{ Sexto mês de IGC } & Sim & 4 & 10,0 & 5 & 23,8 & \multirow{2}{*}{0,159} \\
\hline & & Não & 36 & 90,0 & 16 & 76,2 & \\
\hline
\end{tabular}


Alves FN, Wolkers PCB, Ferreira DMLM, et al. 5

Tabela 2 - Características clínicas na alta hospitalar e durante o acompanhamento ambulatorial dos RNPTMBP incluídos no estudo

\begin{tabular}{|c|c|c|c|c|c|c|c|}
\hline Variáveis & Momentos & Ocorrência & $\begin{array}{c}n \\
\text { (GCCo) }\end{array}$ & $\begin{array}{l}\text { Porcentagem } \\
\text { (GCCo) }\end{array}$ & $\begin{array}{c}n \\
\text { (GCCa) }\end{array}$ & $\begin{array}{l}\text { Porcentagem } \\
\text { (GCCa) }\end{array}$ & p-valor \\
\hline \multirow{8}{*}{$\begin{array}{c}\text { Dieta } \\
\text { exclusivamente } \\
\text { por fórmula láctea }\end{array}$} & \multirow{2}{*}{ Alta } & Sim & 28 & 48,1 & 4 & 11,1 & \multirow{2}{*}{0,000} \\
\hline & & Não & 29 & 50,9 & 32 & 88,9 & \\
\hline & \multirow{2}{*}{ Primeira consulta } & Sim & 25 & 47,2 & 8 & 34,8 & \multirow{2}{*}{0,314} \\
\hline & & Não & 28 & 52,8 & 15 & 65,2 & \\
\hline & \multirow{2}{*}{ Quarto mês de IGC } & Sim & 31 & 77,5 & 13 & 50,0 & \multirow{2}{*}{0,021} \\
\hline & & Não & 9 & 22,5 & 13 & 50,0 & \\
\hline & \multirow{2}{*}{ Sexto mês de IGC } & Sim & 36 & 90,0 & 14 & 70,0 & \multirow{2}{*}{0,057} \\
\hline & & Não & 4 & 10,0 & 6 & 30,0 & \\
\hline \multirow{6}{*}{ Reinternação } & \multirow{2}{*}{ Primeira consulta } & Sim & 6 & 11,3 & 0 & 0,0 & \multirow{2}{*}{0,033} \\
\hline & & Não & 47 & 88,7 & 23 & 100,0 & \\
\hline & \multirow{2}{*}{ Quarto mês de IGC } & Sim & 6 & 15,0 & 6 & 22,2 & \multirow{2}{*}{0,453} \\
\hline & & Não & 34 & 85,0 & 21 & 77,8 & \\
\hline & \multirow{2}{*}{ Sexto mês de IGC } & Sim & 4 & 10,0 & 1 & 4,8 & \multirow{2}{*}{0,460} \\
\hline & & Não & 36 & 90,0 & 20 & 95,2 & \\
\hline \multirow{6}{*}{$\begin{array}{l}\text { Comparecimento } \\
\text { às consultas }\end{array}$} & \multirow{2}{*}{ Primeira consulta } & $\operatorname{Sim}$ & 53 & 93,0 & 23 & 63,9 & \multirow{2}{*}{0,000} \\
\hline & & Não & 4 & 7,0 & 13 & 33,1 & \\
\hline & \multirow{2}{*}{ Quarto mês de IGC } & Sim & 40 & 70,2 & 27 & 75,0 & \multirow{2}{*}{0,612} \\
\hline & & Não & 17 & 29,8 & 9 & 25,0 & \\
\hline & \multirow{2}{*}{ Sexto mês de IGC } & Sim & 40 & 70,2 & 21 & 58,3 & \multirow{2}{*}{0,244} \\
\hline & & Não & 17 & 29,8 & 15 & 41,7 & \\
\hline
\end{tabular}

Fonte: Elaborado pelos autores (2021).

Legenda - IGC: idade gestacional corrigida; AME: aleitamento materno exclusivo, AMM: aleitamento materno misto.

A Tabela 3 mostra, por meio de regressão logística, que a segunda e terceira etapas do MC aumentaram em 11,2 vezes a chance de o RNPT estar em AME, no momento da alta e, em 14,4 vezes de permanecer em AME, na primeira consulta de acompanhamento ambulatorial.

Tabela 3 - Análise de regressão logística quanto à probabilidade de alimentar, por meio de aleitamento materno exclusivo, após ser assistido pelo Método Canguru em diferentes momentos

\begin{tabular}{lcccc}
\hline Momentos & $\begin{array}{c}\text { OR } \\
\text { Sem ajuste }\end{array}$ & p-valor & $\begin{array}{c}\text { OR } \\
\text { Ajustado * }\end{array}$ & p-valor \\
\hline Alta & 11,2 & 0,03 & 17,3 & 0,02 \\
Primeira consulta & 14,4 & 0,02 & 7,3 & 0,09 \\
Quarto mês de IGC & 1,0 & 0,99 & 1,0 & 0,99 \\
Sexto mês de IGC & 1,0 & 0,99 & 1,0 & 0,99 \\
\hline
\end{tabular}

Fonte: Elaborado pelos autores (2021).

Legenda - IGC: idade gestacional corrigida; OR: Odds Ratio; *: Odds Ratio ajustado para peso de alta e idade gestacional de alta.

\section{DISCUSSÃO}

Os RNPT que passaram um período de internação na UCINCa apresentaram maiores taxas de AME na alta hospitalar, na primeira consulta de acompanhamento ambulatorial e no quarto mês de IGC, quando comparado àqueles que permaneceram nas UCINCO.

Ser assistido pela segunda e terceira etapas do $M C$ aumentou em 11,2 vezes $(p=0,02)$ a probabilidade de estar em AME no momento da alta hospitalar e 14,4 vezes $(p=0,01)$ de permanecer em AME na primeira consulta de acompanhamento ambulatorial. Estudos prévios realizados no Brasil, avaliando o MC, também, observaram aumento nas taxas de aleitamento materno ${ }^{(9)}$. Resultados semelhantes foram observados, em uma revisão sistemática com metanálise que, apesar de analisar somente a posição canguru, sugeriu que realizar o contato pele a pele aumenta em $50 \%$ a probabilidade de AME na alta hospitalar ${ }^{(10)}$.

Observamos também menor peso associado ao grupo GCCa no momento da alta hospitalar. Entretanto, no quarto e sexto meses de IGC, ambos os grupos apresentaram resultados semelhantes. Apesar de menores médias de peso estarem relacionadas ao grupo GCCa, esses valores ainda permaneceram elevados, visto que o manual técnico do Ministério da Saúde sugere que, para a alta hospitalar, o peso mínimo seja de $1600 \mathrm{~g}$ e, para a alta da terceira etapa, peso mínimo de $2500 g^{(4)}$.

Em estudo que avaliou os resultados neonatais do MC no Brasil, comparando 16 unidades que possuíam ou não a segunda e terceira etapas do método, foram incluídos 958 RNPT, com peso de nascimento de $500 \mathrm{~g}$ a $1.749 \mathrm{~g}$. 
Foi observado que o peso $(p=0,012)$, comprimento $(p=0,039)$ e perímetro cefálico $(p=$ $0,006)$, com 36 semanas de idade gestacional corrigida, foram menores nas unidades canguru, apesar de evidenciarem aumento nas taxas de aleitamento materno ${ }^{(11)}$. Estudos prévios que avaliaram RNPT observaram resultados favoráveis ao $M C$ relacionados ao ganho ponderal ${ }^{(10)}$ e ao percentual de velocidade de ganho de peso na terceira etapa, quando comparado à primeira e segunda etapas do método ${ }^{(12)}$.

Não observamos diferença no tempo médio de internação, tampouco na IGC, no momento da alta hospitalar. Essas variáveis, juntamente com as médias de peso no momento da alta, reforçam a dificuldade e a insegurança por parte das equipes de saúde para alta hospitalar desses RNPTMBP. Esse dado é preocupante, uma vez que quanto maior o tempo de internação, maiores as taxas de desmame ${ }^{(13-14)}$, complicações como sepse tardia(15), além de elevados custos hospitalares ${ }^{(16)}$. Diferente do observado em nossos resultados, estudos internacionais que avaliaram a posição canguru encontraram associação entre o menor tempo de internação e a realização do contato pele a pele ${ }^{(17-18)}$.

Taxas superiores de reinternação foram apontadas no grupo GCCo, no período correspondente ao pós-alta e na primeira consulta de acompanhamento ambulatorial. Poucos estudos brasileiros abordaram essa temática. Revisão sistemática com metanálise evidenciou redução nas taxas de infecção grave/sepse no acompanhamento, após a alta hospitalar, associada à posição canguru ${ }^{(19)}$. Verifica-se também que as taxas de reinternação, principalmente nos primeiros seis meses de vida, podem estar associadas ao desmame precoce e que o aleitamento materno apresenta um fator protetor para reinternações ${ }^{(20)}$.

Os resultados deste estudo demonstraram, ainda, maior número de ausências na primeira consulta no ambulatório de seguimento no grupo GCCa. Esse resultado pode ser explicado pelo fato de existirem mais consultas, nas primeiras semanas após a alta hospitalar, para os RNPT acompanhados na terceira etapa do MC (em média três consultas/semana), o que pode impactar economicamente, gerando gastos com transporte para famílias de menor nível socioeconômico ${ }^{(21)}$, já que a articulação entre o hospital e a atenção primária à saúde, apesar de recomendada ${ }^{(2)}$, ainda é pouco estabelecida ${ }^{(22)}$, especialmente, em grandes cidades. Outra hipótese a ser levantada seria que o acompanhamento mais intenso da díade mãe-filho, durante a terceira etapa do $M C$, determinaria uma sensação de maior confiança materna quanto à saúde e bem-estar do filho e maior capacidade de reconhecimento de sinais de piora. No entanto são necessários outros estudos que abordem mais profundamente esse aspecto.

Associações importantes relacionadas ao empoderamento materno e ao $\mathrm{MC}$ ainda permanecem pouco investigadas ${ }^{(23)}$. $\mathrm{O}$ aumento da segurança e da habilidade da mãe quanto aos cuidados com o RNPTMBP, pilares propostos pelo $M C$, parecem ter sido características importantes que favoreceram o grupo GCCa. Mães mais confiantes, cientes quanto às necessidades do filho, aptas a reconhecer sinais de comunicação de alerta relacionados ao estado físico, também, podem contribuir para melhores desfechos clínicos. No entanto estudos que comprovem essas hipóteses ainda são necessários.

Entre as ações realizadas pela equipe de enfermagem, destacamos então a necessidade de maior empoderamento e protagonismo da classe, enquanto membro da equipe multidisciplinar com maior possibilidade de estreitar as relações equipe-famílias, o que pode influenciar positivamente à adesão dos pais aos cuidados prescritos. Instituir e executar práticas baseadas em evidências impactam diretamente em desfechos clínicos positivos, tanto no momento da alta hospitalar quanto no acompanhamento ambulatorial.

\section{CONCLUSÕES}

Concluímos que as segunda e terceira etapas do $M C$ impactam positivamente na prática e manutenção do AME. Além disso, foram observados menores índices de reinternação no GCCa até o sexto mês de IGC dos RNPTMBP, apesar de o ganho ponderal ter sido semelhante, em ambos os grupos, após a alta hospitalar.

Como limitações deste estudo, destacamos a fonte dos dados ser obtida por meio de prontuário, visto que há o risco de que o registro não seja realizado corretamente. Além disso, ocorreram perdas no número de pacientes acompanhados, principalmente após a alta hospitalar, não sendo possível a busca ativa por tratar-se de estudo retrospectivo. O tipo de estudo retrospectivo impossibilitou também a avaliação de outras variáveis importantes, como tempo de realização da posição canguru, impacto do $\mathrm{MC}$ no vínculo entre mãe e filho e confiança materna. 
Faz-se necessário maior incentivo à participação ativa do profissional enfermeiro, em todos os processos do cuidar desses neonatos e seus pais, em consonância com os demais profissionais de saúde que promovem a assistência neonatal, para que a implementação do MC seja realizada de maneira ativa, em todas as etapas, com vista à promoção do cuidado integral, equânime e ampliado.

\section{REFERÊNCIAS}

1 - Silva NR, Oliveira J, Berenguer A, Graça AM, Abrantes $\mathrm{M}$, Monis C. Morbidity in Prematurity Associated with Fetal Growth Restriction: Experience of a Tertiary Care Center. Acta Med Port. 2018;31(11): 648-55. DOI: 10.20344/amp.9599

2 - Huff K, Rose RS, Engle WA. Late preterm infants: Morbidities, mortality, and management recommendations. Pediatr Clin North Am. 2019;66(2):387-402.

DOI:

10.1016/j.pcl.2018.12.008

3 - Quaresma ME, Almeida AC, Méio MDB, Lopes JMA, Peixoto MVM. Factors associated with hospitalization during neonatal period. J Pediatr. 2018;94(4):390-8.

DOI: 10.1016/j.jped.2017.07.011

4 - Brasil. Ministério da Saúde. Atenção humanizada ao recém-nascido de baixo peso: Método Canguru: Manual técnico. 3a ed. Brasília: Ministério da Saúde; 2017.

5 - Casper C, Sarapuk I, Pavlyshyn H. Regular and prolonged skin-to-skin contact improves shortterm outcomes for very preterm infants: A dosedependent intervention. Arch Pediatr. 2018;25(8):469-75.

DOI:

\subsection{6/j.arcped.2018.09.008}

6 - Aldana AAC, Tessier R, Charpak N, Tarabulsy G. Randomised controlled trial on the impact of kinesthetic stimulation on early somatic growth of preterm infants in Kangaroo position. Acta Paediatr. 2019;108(7):1230-6. DOI: 10.1111/apa.14675

7 - Charpak N, Ruiz-Pelaez JG, Figueroa de CZ, Charpak Y. Kangaroo mother versus traditional care for newborn infants $</=2000$ grams: A randomized, controlled trial. Pediatrics 1997;100(4):682-8. DOI: 10.1542/peds.100.4.682

8 - Yismaw AE, Gelagay AA, Sisay MM. Survival and predictors among preterm neonates admitted at
University of Gondar comprehensive specialized hospital neonatal intensive care unit, Northwest Ethiopia. Ital J Pediatr. 2019;45(1):1-11. DOI: 10.1186/s13052-018-0597-3

9 - Basso CSD, Arroyo MAS, Saes MABF, Beani L, Maia $A B$, Lourenção $L G$. Breastfeeding rate and speech-language therapy in the Kangaroo Method. Rev CEFAC 2019;21(5):1-9. DOI: 10.1590/19820216/201921511719

10 - Boundy EO, Dastjerdi R, Spiegelman D, Fawsi WW, Missmer SA, Lieberman E, et al. Kangaroo mother care and neonatal outcomes: A metaanalysis. Pediatrics 2016;137(1): 1-18. DOI: 10.1542/peds.2015-2238

11 - Lamy Filho F, Silva AA, Lamy ZC, Gomes MA, Moreira ME. Evaluation of the neonatal outcomes of the kangaroo mother method in Brazil. J Pediatr. 2008;84(5):428-35. DOI: 10.2223/JPED.1821

12 - Souza AKCM, Tavares ACM, Carvalho DGL, Araújo VC. Ganho de peso em recém-nascidos submetidos ao contato pele a pele. Rev CEFAC 2018;20(1):53-60. DOI: 10.1590/1982$\underline{021620182018317}$

13 - Nobre RG, Azevedo DV, Almeida PC, Almeida NM, Feitosa FE. Weight-gain velocity in newborn infants managed with the kangaroo method and associated variables. Matern Child Health J 2017;21(1):128-35. DOI: 10.1007/s10995-0162101-2

14 - Maia C, Brandao R, Roncalli A, Maranhao H. Length of stay in a neonatal intensive care unit and its association with low rates of exclusive breastfeeding in very low birth weight infants. J Matern Fetal Neonatal Med. 2011;24(6):774-7. DOI: $\underline{10.3109 / 14767058.2010 .520046}$

15 - Balaminut T, Sousa MI, Gomes ALM, Christoffel MM, Leite AM, Scochi CGS. Aleitamento materno em prematuros egressos de hospitais amigos da criança do Sudeste. Rev Eletrônica Enferm. 2018 [citado em 28 nov 2020]; 20:1-14. Acesso em:

https://revistas.ufg.br/fen/article/view/50963

16 - Sousa DS, Sousa Júnior AS, Santos ADR, Melo EV, Lima SO, Almeida-Santos MA, et al. Morbidity in extreme low birth weight newborns hospitalized in a high risk public maternity. Rev Bras Saúde Mater Infant. 2018;17(1):139-47. DOI: 10.1590/1806-93042017000100008

17 - Cavallo MC, Gugistti A, Gerzeli S, Bsrbieri D, Zanini R. Cost of care and social consequences of 
very low birth weight infants without prematurerelated morbidites in Italy. Ital J Pediatr. 2015;41(59):1-12. DOI: 10.1186/s13052-0150165-z

18 - Cattaneo A, Davanzo R, Worku B, Surjono A, Echeverria $M$, Bedri $A$, et al. Kangaroo mother care for low birth weight infants: a randomised controlled trial in different settings. Acta Paediatr. 1998;87:976-85. DOI: $\quad$ 10.1111/j.16512227.1998.tb01769

19 - Conde-Agudelo A, Díaz-Rossello JL. Kangaroo mother care to reduce morbidity and mortality in low birthweight infants. Cochrane Database Syst Rev. 2014; 22(4):CD002771. DOI: 10.1002/14651858.CD002771.pub3

20 - Silva TR, Rossetto EG, Souza SNDH, Baena JA. $A$ incidência de reinternações entre prematuros de muito baixo peso e suas associações. Rev Varia Scientia 2015;2(1):119-29. DOI: $\underline{10.48075 / v s c s . v 1 i 2.12912}$

21 - Araújo CL, Rios CTF, Santos MH, Gonçalves APF. Método Mãe Canguru: Uma investigação da prática domiciliar. Cienc Saude Coletiva 2010;15(1):301-7. DOI: 10.1590/S1413$\underline{81232010000100035}$

22 - Reichert APS, Soares AR, Bezerra ICS, Guedes ATA, Pedrosa RKB, Vieira DS. Terceira etapa do método canguru: Experiência de mães e profissionais da atenção primária. Esc Anna Nery 2021;25(1): 1-7. DOI: 10.1590/2177-9465-ean2020-0077

23 - Braga DF, Machado MM, Bosi ML. Achieving exclusive breastfeeding of premature babies: The perceptions and experience of women from public health services. Rev Nutr 2008;21(3):293-302. DOI: 10.1590/S1415-52732008000300004
Nota: Artigo oriundo da dissertação intitulada "Impacto da segunda e terceira etapas do Método Canguru nas variáveis clínicas neonatais: do nascimento ao sexto mês de idade gestacional corrigida", apresentada ao Programa de PósGraduação em Ciências da Saúde da Universidade Federal de Uberlândia.

Recebido em: 26/01/2021

Aprovado em: 03/05/2021 